\title{
VARIATIONAL METHODS AND PARTIAL DIFFERENTIAL EQUATIONS IN CARDIAC IMAGE ANALYSIS
}

\author{
Nikos Paragios \\ C.E.R.T.I.S \\ Ecole Nationale des Ponts et Chaussees \\ 19, rue Alfred Nobel, Cite Descartes \\ Champs sur Marne, 77455 Cedex 2, France
}

\begin{abstract}
Cardio-vascular diseases is a major cause of deaths worldwide. Early diagnosis is quite often associated with more efficient treatment leading to a significant decrease of the mortality rate.

In this paper, we present tools developed to procces different cardiac modalities over the past years with aim to faciliate/automate computer aided diagnosis. In particular we present algorithms and advanced techniques for the segmentation of the left ventrile for magentic resonance and echocardiographic imaging. Furthermore, to account for user interaction, we extent our method towards user-aided boundary delineation where the user has the ability to correct local discrepancies of automated results.
\end{abstract}

\section{INTRODUCTION}

Computer aided diagnosis is a growing application domain of medical image analysis. Segmentation of cardiac structures is used to assist physicians in various states of treatment of cardiovascular diseases.

Identifying the heart chambers, the endocardium and the epicardium is a powerful diagnostic tool. In particular the detection, segmentation and tracking of the left ventricle is of great importance because it pumps oxygenated blood out to distant tissue in the entire body. Furthermore, measuring the ventricular blood volume, wall mass, wall motion and the wall thickening properties over various stages of the cardiac cycle are components with strong diagnostic power.

Segmentation is an ill-posed problem. Pose and reflection properties of the object, noise from the acquisition devices are some of the factors that can interfere with the process. Medical imaging is a bounded area regarding the above conditions. The clinical user can control the acquisition process while sensor perturbations can be considered known. Last, but not least, the physical entities to be recov-

This work was carried out during the affiliation of Prof. Paragios with Siemens Corporate Research, Princeton, NJ 08540. ered are constrained to follow known topology with certain degree of variation.

In this paper we propose a level set framework for shapedriven knowledge-based segmentation and user-aided boundary delineation that is parameter free, implicit and intrinsic. Prior shape knowledge is represented using a probabilistic level set distance map and global shape consistency is inherited to the process through a similarity registration of the evolving interface to the prior model. Visual evidence is integrated through a boundary and a region-based segmentation module while internal smoothness constraints are also imposed. Last, but not least user interaction is transformed to propagation constraints that force the solution to respect the user preferences. Related segmentation techniques with our approach can be found in [1].

The reminder of this paper is organized as follows; In section 2 we introduce the application context and the level set method. Prior shape knowledge is introduced in section 3 while in section 4 we present a technique to account for user-interaction. Discussion is part of section 5.

\section{LEVEL SET DATA-DRIVEN SEGMENTATION}

The segmentation of the left ventricle can be viewed as a bimodal frame partition problem. One would like to separate the endocardium from the background. We address this partition by considering a curve propagation approach. Visual (boundary and regional) terms, prior shape knowledge and internal constraints are used to derive an automated solution for detection and segmentation of the left ventricle.

The level set method [2,3] is an emerging technique for tracking moving interfaces. To this end, based on a motion equation that dictates the propagation of a closed structure, one can construct a structure of a higher dimension $[\phi]$ and define a corresponding flow such that its zero level set yields always to the position of the input structure. A step further is to consider the definition of the problem and the objective function [4] directly on the space of level set representations. 
Towards this end, one can define the approximations of Dirac and Heaviside [4] distributions;

$$
\begin{gathered}
\delta_{a}(\phi)= \begin{cases}0 & ,|\phi|>\alpha \\
\frac{1}{2 \alpha}\left(1+\cos \left(\frac{\pi \phi}{a}\right)\right) & ,|\phi|<\alpha\end{cases} \\
H_{\alpha}(\phi)= \begin{cases}1 & , \phi>\alpha \\
0 & , \phi<-\alpha \\
\frac{1}{2}\left(1+\frac{\phi}{\alpha}+\frac{1}{\pi} \sin \left(\frac{\pi \phi}{a}\right)\right) & ,|\phi|<\alpha\end{cases}
\end{gathered}
$$

and use them to introduce an image partition objective function.

Boundary attraction as well region-consistency terms can be defined based on an evolving function $\phi$. The geodesic active contour [5,6] can be used for example to perform boundary extraction.

$$
E_{B}(\phi)=\underbrace{\iint_{\Omega} \delta_{\alpha}(\phi) b(|\nabla I|)|\nabla \phi| d \Omega}_{\text {boundary module }}
$$

where $b: \mathcal{R}^{+} \rightarrow[0,1]$ is a monotonically decreasing function. The lowest potential of this functional corresponds to a minimal length geodesic curve attracted by the boundaries of the structure of interest.

Regional/global information can improve performance of boundary-based flows [7] that suffer of being sensitive to the initial conditions. The central idea behind this module is to use the evolving interface to define an image partition that is optimal with respect to some grouping criterion. Within level set representations such partition is natural according to the sign of the embedding function. The Heaviside function can be considered to define such partition;

$$
E_{R}(\phi)=\underbrace{\iint_{\Omega} H_{\alpha}(\phi) r_{O}(I) d \Omega}_{\text {endocardium }}+\underbrace{\iint_{\Omega}\left(1-H_{\alpha}(\phi)\right) r_{B}(I) d \Omega}_{\text {background }}
$$

according to some region descriptors functions $r_{O}: \mathcal{R}^{+} \rightarrow$ $[0,1], r_{B}: \mathcal{R}^{+} \rightarrow[0,1]$ that are monotonically decreasing functions. Such descriptors measure the quality of matching between the observed image and the expected regional properties of the structure of interest and the background.

Integration of the boundary and the region-driven term can be considered to perform segmentation [7], namely the geodesic active region model. In the absence of noise, occlusions and corrupted visual information, such method can deal with local deformations. On the other hand, it cannot account for prior shape knowledge, deal with noisy, corrupted and occluded data.

\section{PRIOR SHAPE KNOWLEDGE}

Shape-driven constraints were considered within the propagation of curves in various ways. To this end, one has first to select an appropriate shape representation when introducing such constraints. Moreover, the extraction of an optimal set of parameters able to describe these constraints is to be done given a set of training examples. We consider a pixelwise stochastic level set representation [8] to encode prior knowledge;

$$
p_{M,\{x, y\}}(\phi)=\frac{1}{\sqrt{2 \pi} \sigma_{M}(x, y)} e^{-\frac{\left(\phi-\phi_{M}(x, y)\right)^{2}}{2 \sigma_{M}^{2}(x, y)}}
$$

defined in the pixel level that consists of two unknown variables;

- The shape image $\phi_{M}$,

- The local degrees of variability image $\sigma_{M}$.

Distance transforms are used as embedding function in the definition of $\phi_{M}$. Such prior model also consists of a variability image that describes the confidence of the prior model. In areas where important local deformations are plausible high variability estimates are present. Variational principles according to the maximum likelihood criterion between the model and a training set are used to determine the function $\Phi_{M}$ and the variability estimates $\sigma_{M}$ [8]. This model can be used within the segmentation process to enforce global shape consistency.

Let $\phi$ be a level set representation to which we would like to introduce a global similarity invariant shape constraint according to the model $\phi_{M}$. We assume that $\phi$ is part of the family of shapes that consists of all possible similarity transformations of the model. Introducing such constraint can be done by updating locally the evolving representation to meet the model properties; optimal local match. Correspondence is determined through a similarity registration.

Thus, given the current state $\phi$, we assume the existence of an ideal transformation $A$ between the evolving representation and the shape model. In order to better account for the nature of the structure of interest, we assume that the optimal registration corresponds to the maximum likelihood between the representation and the model;

$$
\begin{aligned}
& (x, y) \rightarrow A(x, y) \\
& \max _{x, y}\left\{p_{M, A(x, y)}(s \phi(x, y))\right\} \forall(x, y): H_{\alpha}(\phi(x, y)) \geq 0
\end{aligned}
$$

where $s$ is the scale factor of the registration model. Level Set Representations with distance transforms as embedding function are invariant to translation and rotation but not to scale variations [9]. Their values are scaled accordingly. Consequently scale appears as a multiplicative factor in the matching process. Solving segmentation/registration now is equivalent with finding a representation $\phi$ and a global registration model $A$;

$$
E_{M}(\phi, A)=\iint_{\Omega} H_{\alpha}(\phi)\left[\log \left(\sigma_{M}(A)\right)+\frac{\left(s \phi-\phi_{M}(A)\right)^{2}}{2 \sigma_{M}^{2}(A)}\right]
$$

This functional consists of two unknown variables; (i) a level set representation that is optimal when it becomes a 
similarity transformation of the prior model, (ii) a transformation (registration) between the evolving current representation and the model. This term is defined in a qualitative manner; model parts with low variability are more significant than the ones that undergo important local deformations.

One can integrate this module with the previously defined visual driven terms

$$
E(\phi, A)=\beta_{1} E_{B}(\phi)+\beta_{2} E_{R}(\phi)+E_{M}(\phi, A)
$$

where $\beta_{1}, \beta_{2}$ are blending parameters, leading to a datadriven segmentation approach that privileges certain prior knowledge on the structure of interest.

\section{USER-INTERACTION}

Segmentation techniques often require local corrections in particular when the visual information does not support the user-preferred solution. User interaction is a common technique to address this issue. One can consider the case of ultrasonic images. The low signal-to-noise ratio can lead to segmentation discrepancies. Correcting these results will take significantly lesser time than the complete hand drawing of the cardiac contours, which is the standard procedure.

We consider the following form of interaction: a single control point that is used to correct local discrepancies. In order to derive shape constraints within a level set framework, we use linear or quadratic interpolation to converted the user edits into closed structures (shapes).

Quadratic interpolation aims at finding the value of a function at an unknown intermediate point given three data points. That is equivalent with fitting a parabola to the three data points $\left(\left(x_{i-1}, y_{i-1}\right),\left(x_{i}, y_{i}\right)\right.$, and $\left.\left(x_{i+1}, y_{i+1}\right)\right)$

$$
y=A x^{2}+B x+C
$$

where $\mathrm{A}, \mathrm{B}$, and $\mathrm{C}$ are unknowns to be recovered. The simplest method to recover these parameters is using the determinants. Then, the user would like to force the solution to go through such a problematic segment. Within our approach, a core point $(\hat{p})$ provided by the user and smoothness constraints on the solution are used to correct such local discrepancies.

One can consider improving the solution locally by replacing a small segment of the actual solution with the interactive part. Therefore, given a point $(\hat{p})$ the curve points that lie within a distance $d^{1}$ are considered. The use of distance maps as embedding function for the level set representations, provide a straightforward manner to determine these points ${ }^{2}$

$$
\mathcal{N}_{\hat{p}}=\left\{p_{i} \in \Omega:|\phi(p)-d|<\delta\right\}
$$

\footnotetext{
${ }^{1}$ This distance should be greater than the minimum distance between the curve and the control point; $d=D(\hat{p}, \partial \mathcal{R}(t))+\epsilon$.

${ }^{2}$ The assumption that the control point lies on the interior of the data-driven solution has been considered within this condition to recover these points. On can easily modify the condition as follows $\mathcal{N}_{\hat{p}}=$
}

where $\delta \leftarrow 0$. For convex shapes and control points that lie on the object, it can be proved that $\mathcal{N}_{\hat{p}}$ consists of two points (for a reasonable small selection for $d$ ). Such assumption does not hold for any shape. Therefore, more than two points can satisfy the constraint. In order to introduce the interactive segment, only two points of the curve will be considered. We select the ones with maximum angular separation at $(\hat{p})$ :

$$
\begin{gathered}
\left(p_{l}, p_{r}\right): \operatorname{argmax}_{\{i, j\}}\left|p_{i}-p_{j}\right| \\
\left(p_{i}, p_{j}\right) \in \mathcal{N}_{\hat{p}} \times \mathcal{N}_{\hat{p}}
\end{gathered}
$$

The next step is to perform a quadratic interpolation between $\left(p_{l}, p_{r}, \hat{p}\right)$ and determine the interactive segment. Within the level set representations, the current position of the curve is recovered from the zero-level set of the embedding function. The curve points are four-connected (zero-crossings on the image plane), and using a simple connected component rule, we can recover them in a clock-wise order;

$$
\partial \mathcal{R}=\left(p_{0}, \ldots, p_{r}, \ldots, p_{l}, \ldots, p_{N}\right)
$$

where $p_{0}$ is an arbitrary selected point. Towards introducing the interactive segment, one can replace the segment between $p_{l}$ and $p_{r}$ with the one determined by the quadratic interpolation between the control point $\hat{p}$ and $p_{l}$ and $p_{r}$;

$$
\partial \mathcal{U} I=\left(p_{0}, \ldots, p_{r}, q_{1}, \ldots, q_{M}, p_{l}, \ldots, p_{N}\right)
$$

One can embed such a shape in a level set function using the Euclidean distance as embedding function;

$$
\phi_{C}(p)= \begin{cases}0 & , p \in \partial \mathcal{U} I \\ +\mathcal{D}(p, \partial \mathcal{U} I)>0 & , p \in \mathcal{R}_{\mathcal{U I}} \\ -\mathcal{D}(p, \partial \mathcal{U} I)<0 & , p \in\left[\Omega-\mathcal{R}_{\mathcal{U I}}\right]\end{cases}
$$

Such a representation encodes the user edits in a global fashion using the existing solution in areas where user interaction is absent where one should tolerate important deviations from the constraint. We consider the distance between the control point and the image plane as an indicator for the importance of the constraint.

$$
\sigma_{C}(p)=1+|p-\hat{p}|, \quad p \in \Omega
$$

Such a measure will be small for the area around the interactive segment while being significant for the segments that are far from the user edits. The same principle can be used to account for multiple, independent user edits.

The user-edits are taken into account when the evolving level set representation becomes similar to the one derived from the constraint. To this end, we consider the distance between the constraint and the evolving representation $\phi$.

$$
E(\phi)=\iint_{\Omega} H_{\alpha}(\phi)\left(\phi-\phi_{C}\right)^{2} d \Omega
$$

$\overline{\left\{p_{i} \in \Omega:|\phi(p)+d|<\delta\right.}$ to deal with control points that lie on the background. 

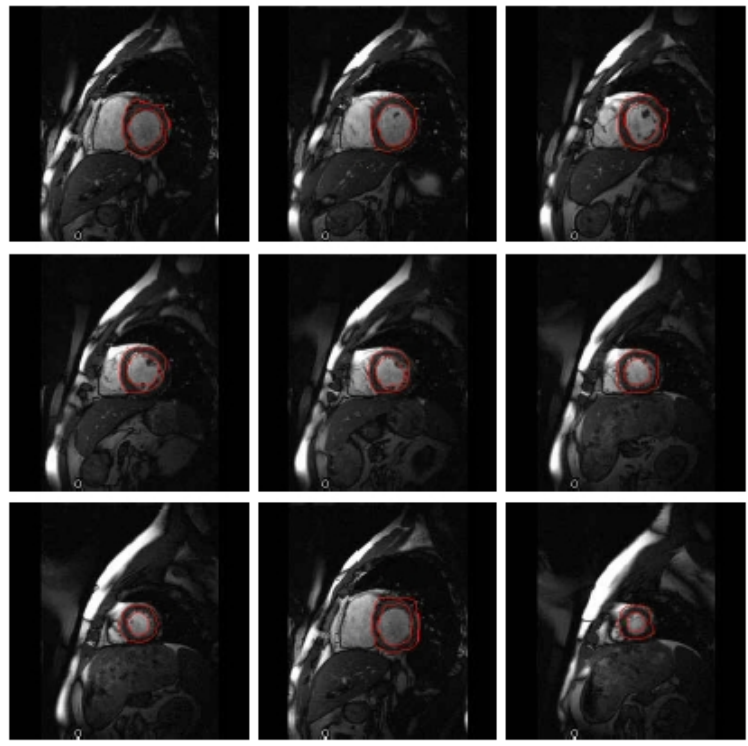

Fig. 1. Visual-information and prior knowledge.

that is equivalent with seeking a curve that goes through the user-defined seed points. This is done by minimizing the distance between the evolving curve and the interactive constraint.

During the model construction, we have considered that the importance of the user-input is determined according to the distance from the control points. It is natural to harder enforce the constraint close to the user seeds while consider the data to guide the segmentation process when there is not input from the user. The distance between the constructed prior and the control points of the constraint can be used to implement such strategy;

$$
E_{I}(\phi)=\iint_{\Omega} H_{\alpha}(\phi) \frac{\left(\phi-\phi_{C}\right)^{2}}{\sigma_{C}^{2}} d \Omega
$$

The user interaction is optimally considered when finding the $\phi$ that corresponds to the lowest potential of the objective function. The calculus of variations within a gradient descent method can be used to determine the optimal flow that forces the evolving curve to respect the user-defined constraints. One can integrate such a constraint with visual terms and domain-specific knowledge.

\section{DISCUSSION}

In this paper we have proposed a shape-driven variational framework for knowledge-based segmentation. Our approach integrates domain specific knowledge, visual information with shape constraints and user-specific knowledge.

Promising experimental results using cardiac [fig. $(1,2)$ ] MRI and ultrasound were obtained. The 3D implementation of our approach is under investigation. Non-parametric shape representations within the space of distance transform
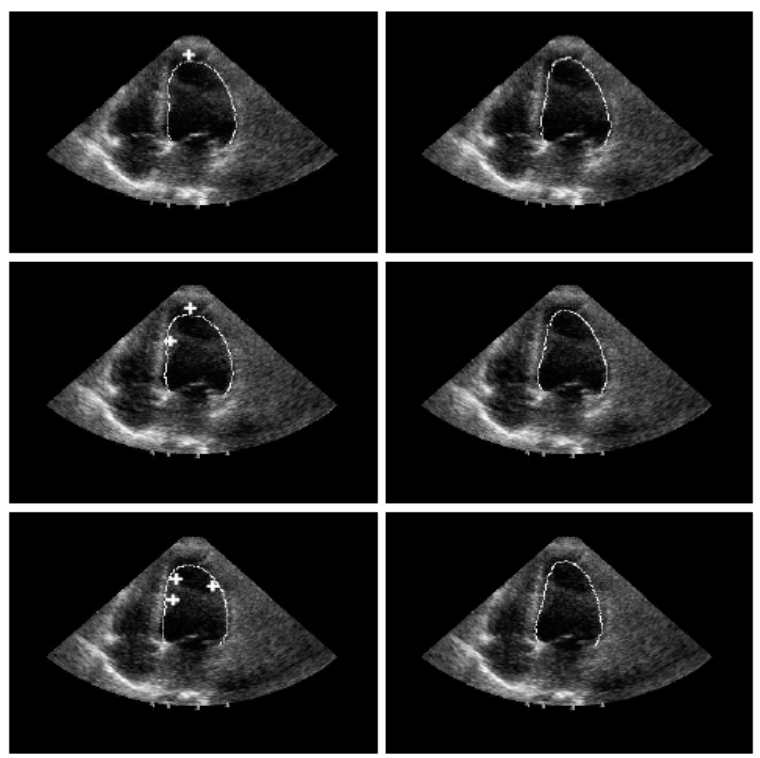

Fig. 2. Visual-information and prior knowledge and user interaction (crosses) (- left - before interaction, - right - after interaction).

is a step forward for our approach [10]. Changes of topology is a strength of level set representations. Our approach can detect single objects with complex topology but cannot recover structures of different topology that are connected.

\section{REFERENCES}

[1] N. Paragios, "A Variational Approach for the Segmentation of the Left Ventricle in Cardiac Image Analysis," IJCV, vol. 50, pp. 345$362,2002$.

[2] S. Osher and J. Sethian, "Fronts propagating with curvaturedependent speed : Algorithms based on the Hamilton-Jacobi formulation," Journal of Computational Physics, vol. 79, pp. 12-49, 1988.

[3] S. Osher and N. Paragios, Geometric Level Set Method in Imaging, Vision and Graphics, Springer, 2003.

[4] H-K. Zhao, T. Chan, B. Merriman, and S. Osher, "A variational Level Set Approach to Multiphase Motion," Journal of Computational Physics, vol. 127, pp. 179-195, 1996.

[5] V. Caselles, R. Kimmel, and G. Sapiro, "Geodesic Active Contours," IJCV, vol. 22, pp. 61-79, 1997.

[6] S. Kichenassamy, A. Kumar, P. Olver, A. Tannenbaum, and A. Yezzi, "Gradient flows and geometric active contour models," in IEEE ICCV, 1995, pp. 810-815.

[7] N. Paragios and R. Deriche, "Geodesic Active Regions: A New Framework to Deal with Frame Partition Problems in Computer Vision," Journal of Visual Communication and Image Representation, vol. 13, pp. 249-268, 2002.

[8] M. Rousson and N. Paragios, "Shape Priors for Level Set Representations," in ECCV, Copenhangen, Denmark, 2002, pp. II:78-93.

[9] N. Paragios, M. Rousson, and V. Ramesh, "Non-Rigid Registration Using Distance Functions," Computer Vision and Image Understanding, vol. 23, pp. 142-165, 2003.

[10] M. Rousson, N. Paragios, and R. Deriche, "Active Shape Models from a Level Set Perspective," Tech. Rep. 4984, I.N.R.I.A., 2003. 